\title{
The effect of vitamin and mineral supplements and health foods on physical endurance and performance
}

By Å. Bruce, Nutrition Laboratory, Swedish National Food Administration, Box 622, S-751 26 Uppsala, Sweden and B. ЕквLом, Department of Physiology III, Karolinska Institute, Stockholm, Sweden and I. Nilsson, The National Corporation of Swedish Pharmacies, Stockholm, Sweden

A nutritionally-adequate diet is one that provides sufficient nutrients and energy to meet metabolic needs for optimal functioning of the body. Optimal nutrition is also necessary for the development and maintenance of top physical performance. Nutritional conditioning, like physical conditioning, is a continuous quest, not something to be practised $\mathrm{I}$ or $2 \mathrm{~d}$ before competition. Sports competitors often experiment with fad diets, protein supplements and megavitamin therapy in order to achieve the 'competitive edge'. There is, however, no conclusive evidence that such regimens improve performance. Nutrition has become a topic of interest, but much of the information is inadequate, inaccurate and filled with misconceptions.

In order to investigate the effects of protein, mineral and vitamin supplementation as well as any positive effects of some health food products, the Swedish Sports Federation appointed an expert group which should:

(1) Evaluate the scientific findings on protein, vitamin and mineral requirements of different physical activities.

(2) Evaluate the different scientific studies on various effects of protein, vitamin and mineral supplementation on physical capacity.

(3) Evaluate the scientific literature on some health-food products and their effects on sport competitors.

The report is entirely in Swedish and was presented in 1982 (Sveriges Riksidrottsförbund, 1982). The present paper is a summarized and updated version of the report. The chapter on protein is excluded and this presentation will mainly concentrate on ascorbic acid, vitamin $\mathrm{E}$ and iron as well as some health-food products.

\section{Vitamins}

The purpose of this presentation is to review the research relevant to vitamin supplementation and human physical performance. The presentation emphasizes their potential physiological roles in relation to exercise metabolism and performance in competitions.

More vitamins are taken in search of better performance and health than any other nutrients. This practice is followed in spite of the fact that very few scientifically-controlled studies have shown vitamin intake in excess of the recommendations to have any beneficial effects.

Many clinical mineral and vitamin deficiencies are characterized by an impaired 
performance. These deficiency states are usually normalized when the appropriate vitamin or mineral is supplemented. In some studies subclinical deficiencies have been identified among the athletes and a positive effect of a supplement is to be supposed. Therefore, it is important to state if the participants initially may have had a subclinical deficiency, their general nutrient situation and if the participants (including the controls) had used any supplements before the study. In well-designed studies the vitamin status of the participants should be analysed both before and during the study. The positive results in some studies can probably be explained by the fact that the vitamin supplementation treated a subclinical deficiency.

Many studies have been performed on training subjects, when positive training effects are to be expected. Improvement due to training may mask any possible benefits that vitamin supplementation may have. It may also complicate the interpretation of the results, since two probably not totally independent variables are studied.

The vitamins are engaged in various physiological processes and many animal studies have shown that extra vitamins may enhance these processes at least on a cellular level. It is, however, doubtful if the results from isolated, well-controlled animal studies or from human experiments in physiological laboratories are applicable to the competing athlete.

The most appropriate investigations ought to be those where results from competitors are compared between two groups of equal athletes given either a supplement or placebo in a double-blind study. Only a few such studies have been published.

Many papers have been written about the effects of vitamin supplementation on sportsmen. Older studies usually report positive effects while studies published during the last ro-1 5 years usually do not find any differences between groups given supplement or placebo. The latter studies usually are better controlled and are better designed than the older studies and the results are evaluated statistically. It is also remarkable that studies from Central and East Europe usually report positive effects which cannot be confirmed by British or American studies.

\section{Ascorbic acid}

Ascorbic acid has several roles in cellular metabolism. It is known to function in the synthesis of collagen, epinephrine and corticoids of the adrenal gland. It also assists in the absorption of $\mathrm{Fe}$ and serves as a powerful antioxidant. These functions may have several implications for physical performance.

In individuals with subclinical ascorbic acid deficiency, supplementation increases the working capacity, physical performance and may improve sporting performances.

Most well-controlled studies have not been able to show any positive effects of extra ascorbic acid on physical capacity. Several of the positive reports are studies from Central and East Europe, where the normal diet previously had a low content of ascorbic acid, while the negative findings are reported from the USA where the 
intake of ascorbic acid is much higher. The availability of fresh fruit and vegetables, particularly during winter-time, is limited. Therefore the normal diets of many athletes in East Europe were deficient in this vitamin (Haymes, 1980). In some studies (Namyslowski, r960) it has even been admitted that a clinical deficiency occurred.

Competition studies. Only two studies on the effect of ascorbic acid on competition results seem to have been published. Rasch et al. (1962) gave half the members of an American college cross-country team $500 \mathrm{mg}$ daily during the competitive season. The remainder of the team received placebo and served as controls. The intake of ascorbic acid had no effect on the mean times of the runners and it was concluded that the normal diet of these individuals included sufficient amounts of ascorbic acid.

Bender \& Nash (1975) seem to be the only workers who recently have studied the effect on competition results of ascorbic acid supplemention. They studied competitive athletic performance and work efficiency of fifteen short-distance and forty long-distance runners who received either $250 \mathrm{mg}$ ascorbic acid/d or a placebo. The performances under competitive conditions were recorded over two complete athletic seasons for two types of events: short, track events where intensive effort is involved for short periods and long, cross country and road running where endurance is involved. The results were expressed as a percentage of the British championship time for the particular event. There were no significant differences between treated and untreated groups and between the two seasons, for either group, before and after treatment.

Other performance studies. In Table $\mathrm{I}$, values are given from five controlled studies which have included various aerobic and anaerobic tests. These studies have been performed on different categories of subjects, from training individuals with low activities to elite athletes.

Only Howald et al. (1975) who examined thirteen athletes on continuous moderate training, found a significant increasing working capacity after 2 weeks of I $\mathrm{g}$ ascorbic acid/d. They found marked improvement in aerobic working capacity and in the biochemical indices that were measured. However, the two treatments were given consecutively (placebo before treatment) and training effect cannot be excluded.

It has been postulated that supplemental doses of ascorbic acid may reduce the severity and morbidity of athletic injuries. Several minor studies have appeared but controlled studies did not indicate any significant difference between groups taking ascorbic acid or a placebo (Gey et al. 1970).

The findings of a number of studies support the contention that the ingestion of ascorbic acid has no beneficial effects in meeting the additional stress imposed by exercise in healthy subjects regardless of their state of training. Ascorbic acid intake even in high doses does not seem to have any influence on the physical capacity. 


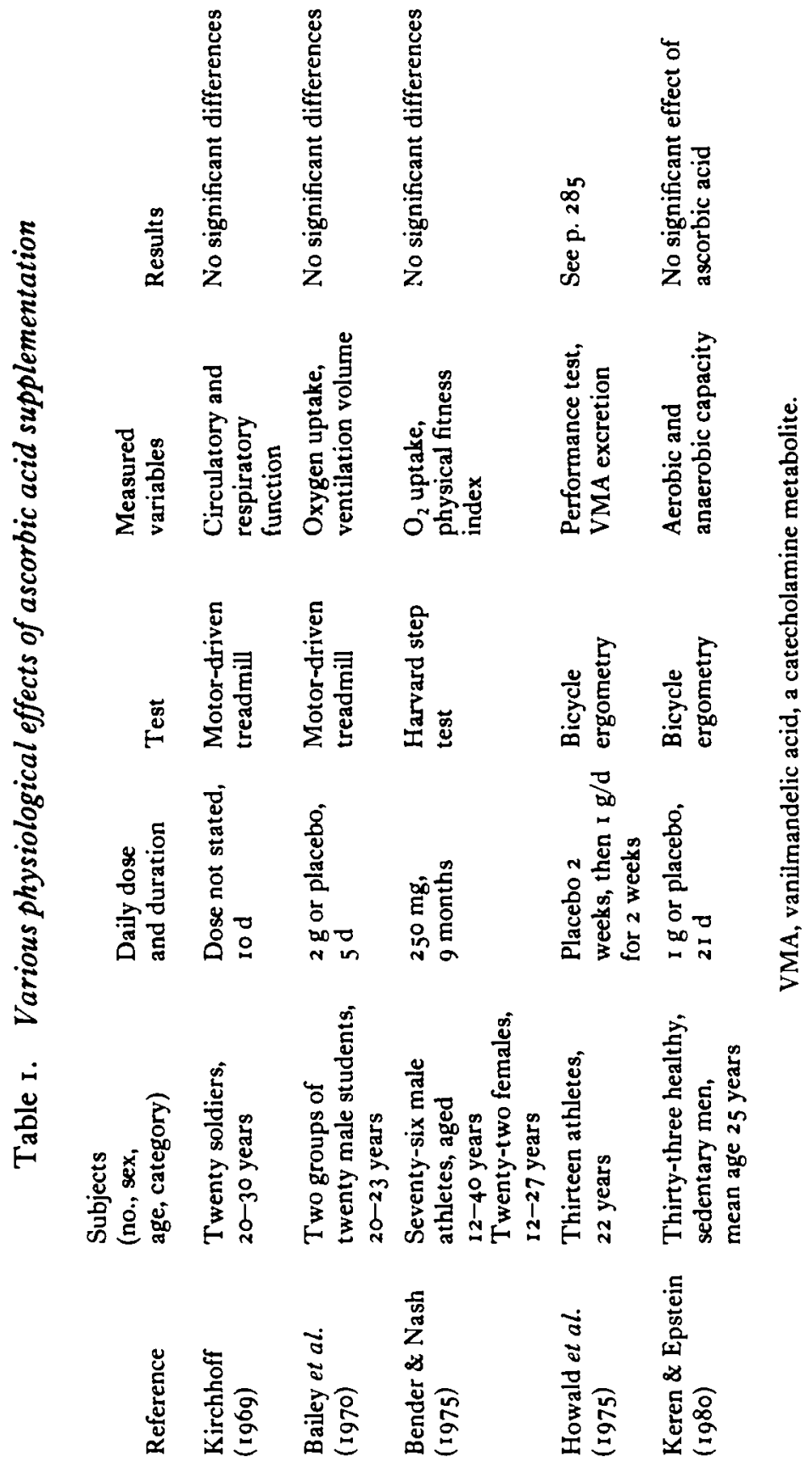




\section{Vitamin E}

Vitamin $\mathrm{E}$ may serve as an antioxidant of polyunsaturated fatty acids. It will thus protect the integrity of the erythrocytes during exercise by preventing oxidation of the phospholipids in the cell membrane. It could also be of some importance for normal muscle function since muscular dystrophy is a prominent symptom in vitamin $\mathrm{E}$ deficiency among some animals.

Competition studies. The only studies on competing athletes have been reported by Sharman et al. (1971, 1976) who first studied boarding-school boys and then trained-swimmers given $400 \mathrm{mg} \alpha$-tocopherol or a placebo in addition to their daily diet. No significant differences as a result of the vitamin $E$ treatment were observed between the two groups in any of the studies. Training, however, significantly improved physical function and performance.

Other studies. Some controlled studies on oxygen uptake, heart beat frequency, working capacity, etc. are summarized in Table 2. Most of these studies did not indicate any significant differences between the supplemented subjects and those who had received the placebo. The only exception is the study by Nagawa $e t$ al. (1968) who studied long-distance runners before the Olympic Games in Mexico City. They observed a positive effect of vitamin $E$ on endurance. Their interpretation is, however, complicated by the fact that both the effects of training, tocopherol supplementation and high-altitude adaptations were studied simultaneously. Only small groups of individuals were studied and it is doubtful whether any statistical evaluation was done.

\section{Fe}

Daily $\mathrm{Fe}$ intake needed to maintain body stores is thought to be achieved with intakes of $10 \mathrm{mg} / \mathrm{d}$ for males and $18 \mathrm{mg} / \mathrm{d}$ for females. Normally, $5-10 \%$ of ingested $\mathrm{Fe}$ is absorbed to replace daily losses, which in men is $\mathrm{I} \mathrm{mg} / \mathrm{d}$ through stool, urine, sloughed skin and nails, and in women is up to $\mathrm{I} \mathrm{mg} / \mathrm{d}$ in menstrual loss.

Fe loss via the sweat is usually considered to be negligible in humans. Vellar (1968), however, has shown that in cases of extreme sweating, as much as $400 \mu \mathrm{g}$ $\mathrm{Fe} / \mathrm{l}$ sweat might be lost. As the sweat production during $\mathrm{I} d$ of endurance training may well amount to $\mathrm{I}-3$ litres, an additional loss of $0.4-1 \cdot 2 \mathrm{mg} \mathrm{Fe} / \mathrm{d}$ is possible. These findings have not been confirmed by $\mathrm{Fe}$ balance studies or studies using isotope techniques, which have indicated only minor losses.

Occasionally, after long-distance runs, haemoglobin and myoglobin have been found in urine. It has been postulated that there is mechanical destruction of fragile older erythrocytes within the circulation and the released $\mathrm{Fe}$ is utilized in the formation of myoglobin and new erythrocytes. Observations of a decrease of haptoglobin concentration in serum as a sign of accelerated intravasal haemolysis is in good agreement with this assumption. However, the magnitude of these losses are usually so small that they are hardly of any importance.

The most important reason for $\mathrm{Fe}$ losses is bleeding. Considering the average $\mathrm{Fe}$ 


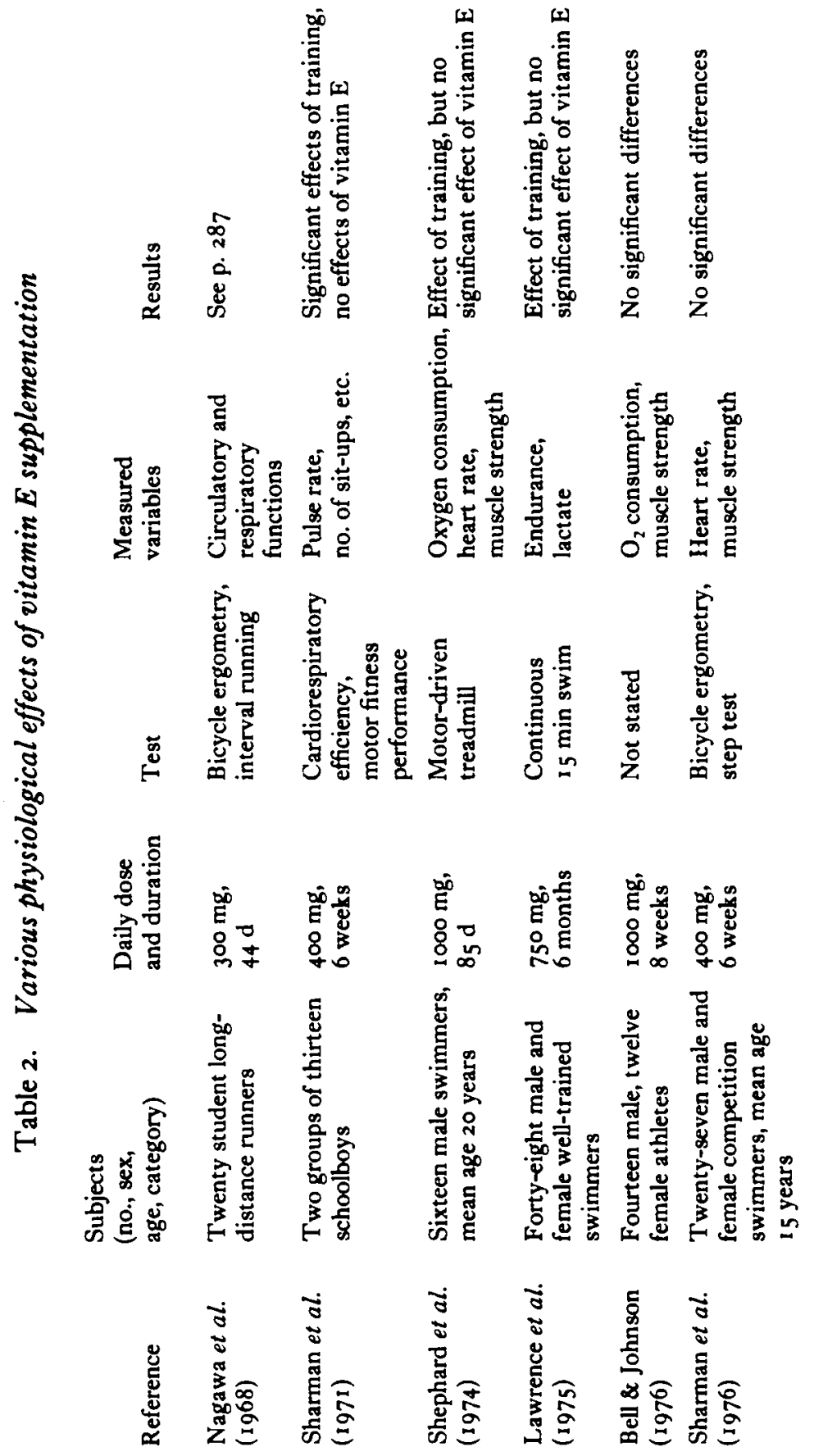


loss of $15 \mathrm{mg}$ caused by the menstrual bleeding, which means an additional daily Fe demand of $0.5^{-1} \mathrm{mg}$, it becomes clear that the female athlete is particularly susceptible to $\mathrm{Fe}$ deficiency (Strauzenberg et al. $198 \mathrm{r}$ ).

Inflammations and injuries often occur in sport and these conditions are often treated with different antiphlogistic drugs. Some of these drugs induce gastrointestinal bleedings with a slow but constant blood loss.

When an untrained subject starts intensive training there is an increased $\mathrm{Fe}$ turnover and subsequent increased $\mathrm{Fe}$ requirements. $\mathrm{Fe}$ is mainly needed for the formation of haemoglobin and myoglobin. If a subject with a blood volume of 4 litres and a haemoglobin concentration of $140 \mathrm{~g} / 1$ increases his blood volume by 0.5 litres (a reasonable increase after 0.5 year of moderately-hard training) and the haemoglobin concentration remains unchanged, the amount of haemoglobin increases by $70 \mathrm{~g}$ corresponding to an $\mathrm{Fe}$ requirement of $240 \mathrm{mg}$. If the $\mathrm{Fe}$ stores initially are low and if the dietary intake is also low, the physical training can decrease the haemoglobin concentration leading to inefficient training results. Normally the body can compensate for the Fe losses by increasing the absorption of dietary Fe. A necessary prerequisite is that the diet contains adequate amounts of Fe.

Fe deficiency can influence the physical performance in several ways. It has been well-documented that depressed haemoglobin levels are associated with impaired $\mathrm{O}_{2}$-carrying capacity in the blood and with a decreased maximum aerobic power. Findings published by a considerable number of investigators (e.g. Strauzenberg et al. 198I) reveal that even a reduction of haemoglobin to $120 \mathrm{~g} / \mathrm{l}$, which is well above the lower limit of the 'normal' range of $110 \mathrm{~g}$ haemoglobin/l, is accompanied by a significant decrease in physical working capacity. Ekblom et al. (1972) reported that a decrease in haemoglobin concentration of $19 \mathrm{~g} / \mathrm{l}$ leads to a reduction in physical working capacity of $29 \%$.

$\mathrm{Fe}$ supplementation of athletes with 'anaemia' increases the physical performance. Systemic Fe deficiency was found by Hunding et al. (1981) in sixty-three of $\mathrm{I}_{3} 3$ joggers and competition runners (thirty-three women and eighty men). Thirteen women and ten men had latent anaemia. Oral Fe therapy (200 mg ferrous sulphate/d) normalized the haemoglobin concentration and improved the transferrin saturation fraction in sixty-one people. The competition runners reported personal records.

Ekblom et al. (1972) have also shown that work performance can be improved almost immediately when the $\mathrm{O}_{2}$-carrying capacity of the blood is simply increased through blood transfusion.

Sports anaemia. It has been known for a long time that well-trained endurance athletes have lower packed cell volume and haemoglobin values than non-athletes but that their total amount of circulating haemoglobin is increased. Three main hypotheses have been advanced (Hallberg \& Magnusson, 1984).

(1) The anaemia may be a favourable adaptation to the increased $\mathrm{O}_{2}$ demands as a lower packed cell volume reduces the peripheral vascular resistance and may in this way lead to a better $\mathrm{O}_{2}$ delivery to the tissues. 
(2) The anaemia may be due to an increased mechanical destruction of erythrocytes, a haemolytic anaemia.

(3) The anaemia may be caused by a lack of $\mathrm{Fe}$, possibly due to increased losses of $\mathrm{Fe}$ in urine and sweat, an Fe-deficiency anaemia.

None of these hypotheses is regarded by Hallberg \& Magnusson (1984) as an important explanation for 'sports anaemia'. Two recent studies have given new insight into this problem.

In the first study, forty-three elite male runners were screened and two groups of subjects were selected for a very extensive study on their Fe metabolism by Magnusson et al. (1984a). One group had values suggesting Fe depletion while the contrasting group gave values indicating good $\mathrm{Fe}$ repletion. The studies comprised a number of analyses and when the values were pooled it was obvious that none of the subjects were truly $\mathrm{Fe}$ deficient.

In a subsequent study a haematological comparison was performed between forty-three middle- and long-distance male runners and ing control men (Magnusson et al. 1984 ). Although the values for packed cell volume, serum $\mathrm{Fe}$ transferrin saturation and serum ferritin were lower in the athletes than in the controls they were not as low as to indicate Fe deficiency. These and other findings strongly supported the conclusion that the lack of Fe had not limited erythropoiesis or the formation of an optimal erythrocyte mass. The changes in the serum Fe and serum ferritin suggesting $\mathrm{Fe}$ deficiency or lowered $\mathrm{Fe}$ stores, could be explained by a shift in Fe metabolism.

Low serum haptoglobin values in most athletes indicated an increased intravascular haemolysis. As the haemoglobin-haptoglobin complex formed is taken up by the hepatocytes, this implies that there is a shift in the erythrocyte catabolism from the reticulo-endothelial system to the hepatocytes. This may explain the paradoxical findings of low serum ferritin concentrations and reduced contents of bone marrow haemosiderin. This is consistent with the observed normal erythropoiesis (see Fig. I).

Hallberg \& Magnusson (1984) also has a complementary explanation. Since these athletes were training for several hours every day, their extraction of $\mathrm{O}_{2}$ from the haemoglobin should be quite high for long periods of time. This will change the conditions in the erythrocytes leading to a shift in the $\mathrm{O}_{2}$-dissociation curve of the haemoglobin to the right. This in turn leads to an impaired binding of $\mathrm{O}_{2}$ to haemoglobin which leads to an increased delivery of $\mathrm{O}_{2}$ to the tissues at any level of $\mathrm{O}_{2}$ tension. The sensor responsible for the regulation of the erythropoietin level will get the same information about the $\mathrm{O}_{2}$-delivery capacity as if the haemoglobin levels had been higher. This leads to a relative decrease in the production of erythropoietin and thus to a setting of the packed cell volume and haemoglobin concentration at a lower level.

The balance of evidence thus indicates that 'sports anaemia' is the result of physiological response to the increased prolonged extraction of $\mathrm{O}_{2}$ from the haemoglobin. The increased intravascular destruction of erythrocytes in 


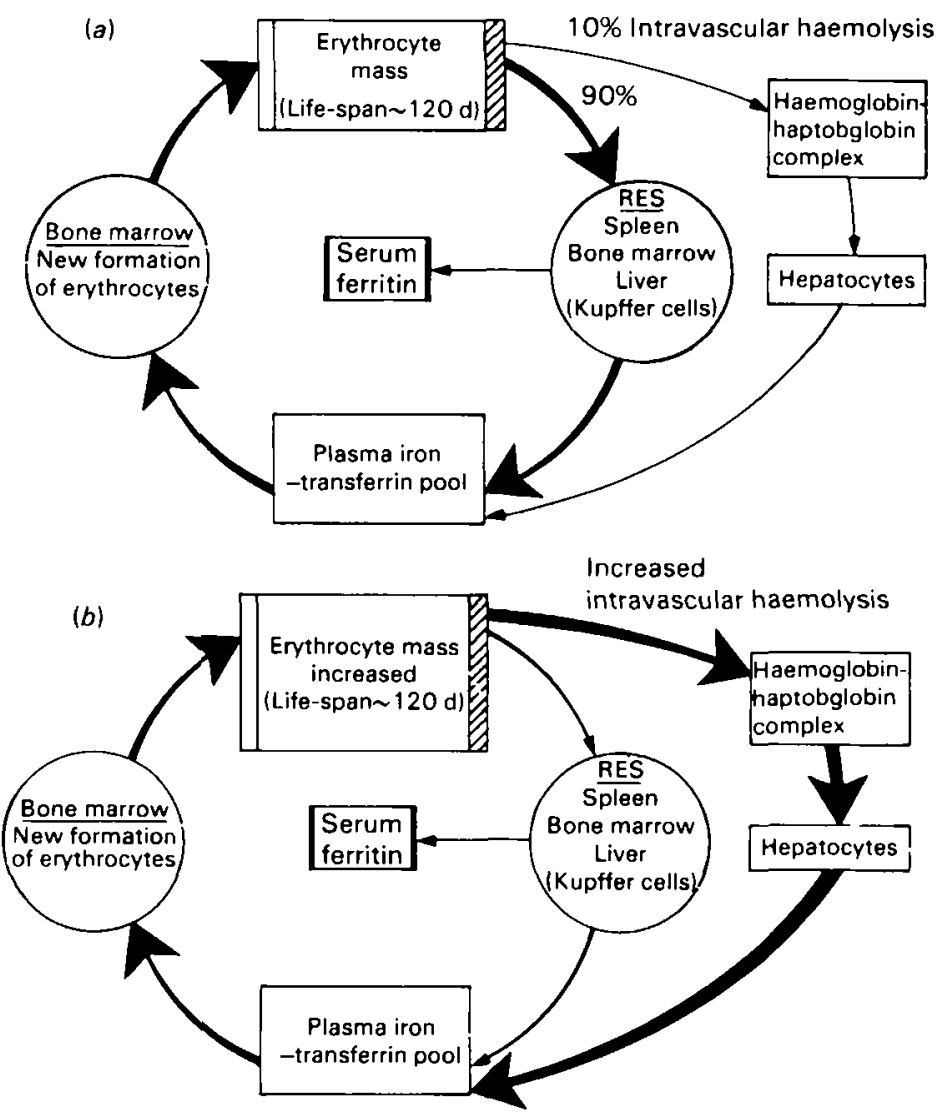

Fig. I. Erythrocyte formation and destruction in $(a)$ normal subjects and $(b)$ runners (Magnusson et $a l .1984 b)$. RES, reticulo-endothelial system.

long-distance runners should also be considered as 'side effects' of the intensive physical training.

'Runners anaemia' is no true anaemia, and is not caused by an Fe deficiency. 'Sports anaemia' is thus no indication for routine Fe supplementation (Hallberg \& Magnusson, 1984).

\section{Ginseng and Russian root}

Some health foods like ginseng, Russian root, pollen and pangamic acid are popular in Sweden among athletes, especially among weight-lifters and body builders. In advertisements intended for athletes they are said to increase endurance and strength.

Ginseng is the root of two plants, Panax ginseng and Panax quinquefolius. Since ancient times Panax ginseng has been cultivated in China and Korea. Panax quinquefolius is cultivated in Japan and in the USA. The shape of the root resembles that of a human being and it is surrounded by mystique. According to the Chinese the root is a panacea, which is supposed to give a long life, increase libido and cure a variety of diseases. The active components of ginseng are 
triterpene saponines, called ginsenosides. More than ten ginsenosides have been isolated and their structures determined.

Animal experiments, e.g. Avakian \& Evonuk (1979), with high doses of pure ginsenosides and root extracts have shown various effects on blood sugar and blood pressure, increased protein synthesis, stimulation of the immune system, reduced glycogen metabolism and increased power of endurance during prolonged exercise. It is still unknown how the ginsenosides work. Certain test results on animals suggest that they act like hormones, that is they influence the suprarenals to release adrenalin. The effects of ginseng on sports' achievements are not well documented. The available literature consists of a few congress reports and extracts from books (Forgo, 1980) which are difficult to interpret.

Adverse effects of ginseng have also been reported. In one study comprising 133 men and women, who were given $3^{-15} \mathrm{~g}$ ginseng extract every day, the test subjects showed adverse reactions like high blood pressure, acne, oedema, diarrhoea and nervousness (Siegel, 1979). Cases of mastalgia and oestrogen-like effects have also been reported (Dukes, I978; Punnonen \& Lukola, I 980).

From the results of animal experiments it can be assumed that ginseng in large doses might interfere with certain functions of the human body. How and at which doses is not yet known. That ginseng should have a stronger positive effect than a placebo on athletic performances has not been confirmed.

Russian root is the root of a bush, Eleuterococcus senticosus, that grows in eastern Sibiria, China and Korea. It belongs to the same family as ginseng (Araliaceae) and is used as a substitute for ginseng. The active components of Russian root are triterpene saponines, called eleuterosides. Animal experiments have shown that the eleuterosides have effects similar to those of ginseng (Dardymov et al. 1978). It has hitherto been impossible to find documentation confirming that Russian root should have a positive effect on athletic performances. The few existing reports on such an effect are not convincing.

\section{Pollen}

Pollen tablets contain an extract of microbiologically-fermented pollen. They are composed of either a water extract or an alcohol extract. Both extracts contain hydrolysed proteins, vitamins, minerals, steroids and sugars. Pollen might thus be considered as a food. It is supposed to contain a pharmacologically-active substance, which has not yet been identified.

Studies have been made in Poland on the effect of pollen tablets on physiological capacity and sporting achievements (Dabrowski, 1980). It is, however, very difficult to draw any conclusion from those studies. Many of them are not double-blind tests and placebo effects can therefore not be excluded. The possibility that the supply of vitamins and minerals cured a deficiency has even been indicated by the authors.

\section{Pangamic acid}

Pangamic acid was originally isolated from apricot stones claimed to contain 
D-glucono dimethyl aminoacetic acid. The Food and Drugs Administration in the USA has examined a large amount of pangamic acid preparations available in the USA. The different brands contained calcium gluconate, glucono dimethyl aminoacetic acid, sodium glutamate, glycine, diisopropylammoniumdichloroacetate and calcium chloride in various concentrations. Pangamic acid is sometimes said to be a mixture of calcium gluconate and dimethylglycine (Herbert, 1979). It is also called vitamin $B_{15}$. However, it is not a vitamin, since it does not cure any known deficiency in man or animal.

In advertisements pangamic acid is said to have a stimulating effect owing to increased uptake of $\mathrm{O}_{2}$ in tissues and cells, and pangamic acid, taken before physical exercise, is supposed to lower the serum concentration of lactate.

Most of the positive studies on the effect of pangamic acid on sports achievements come from the USSR. Karpuchina et al. ( 1967$)$ compared the effect of calcium pangamate with that of methionine or a mixture of calcium gluconate and glycine. According to the English summary, calcium pangamate facilitated the maintenance of a proper blood sugar concentration, reduced serum lactate and the discrepancy between oxidized and reduced gluthathione during physical exercise; results that have not, however, been verified or statistically analysed.

Girandola et al. (1980) have studied the effect of pangamic acid on certain physiological criteria during physical exercise. During a period of 2 weeks eight persons were given $2.4 \mathrm{~g}$ of a mixture of calcium gluconate and $\mathrm{N}, \mathrm{N}$-dimethyl glycine. The maximal $\mathrm{O}_{2}$ consumption, heart beat frequency and level of serum lactate were determined while they were working on an ergometer cycle and compared with the test results of a control group. No significant differences between the two groups were noticed. Since animal experiments have shown that D-glucono methylglycine causes muscular blockade and dimethylglycine is a potential carcinogen (Marshall et al. 1961; Girandola, 1980), the US Food and Drugs Administration consider it unsafe to use pangamic acid.

\section{Conclusions}

Many states of clinical vitamin and mineral deficiencies are characterized by a decreased physical capacity. Several studies have actually demonstrated clinical or subclinical deficiencies in some groups of athletes and vitamin and mineral preparations have raised performance levels in these individuals.

It is doubtful whether the results from well-controlled animal studies or from experiments with humans in a laboratory could actually be applied to the complex situation which competitive sport constitutes. The most revealing investigations ought to be those which compare the results from sport contests between groups of athletes of equal talent who receive as a double-blind a vitamin or mineral preparation. Very few such studies have been conducted and the results have usually been negative.

'Sports anaemia' is stated to occur relatively often in athletes. This phenomenon, however, is considered by others to be a result of a proportionally larger increase in the plasma volume than in the mass of erythrocytes, resulting in 
an apparent decrease in the concentration of haemoglobin. Recent findings indicate that 'sports anaemia' is the result of physiological response to the increased prolonged extraction of $\mathrm{O}_{2}$ from the haemoglobin, leading to a shift to the right of the $\mathrm{O}_{2}$-dissociation curve of haemoglobin. The increased intravascular destruction of erythrocytes forms a haemoglobin-haptoglobin complex which is taken up by the hepatocytes. This implies that there is a shift in the erythrocyte catabolism in these athletes from the reticulo-endothelial system to the hepatocytes. Supplements of $\mathrm{Fe}$ given to athletes with a definite $\mathrm{Fe}$ deficiency anaemia result in an increased physical capacity.

Health-food products, such as ginseng, Russian root and pollen ought to be seen as preparations which can have certain pharmaceutical effects. The sparse scientific reports on the effects of these and similar products on the physical performance in humans present a very mixed picture.

\section{REFERENCES}

Avakian, E. V. \& Evonuk, E. (1979). Planta Medica 36, 43-48.

Bailey, D. A., Carron, A. V., Teece, R. G. \& Wehner, H. (1970). International Yournal of Vitamin Research 40, 436-44I.

Bell, R. D. \& Johnson, G. (1976). Sportarzt und Sportmedizin 27, 178-181.

Bender, A. E. \& Nash, A. H. (1975). Plant Foods for Man $\mathrm{x}, 217^{-2} 3^{1}$.

Dabrowski, I. (1980). Dissertation. Warsaw.

Dardymov, I. V., Khasina, E. I. \& Bezdetko, G. N. (1978). Plant Resources 14, 1-5.

Dukes, M. N. G. (1978). British Medical fournal i, 1621 .

Ekblom, B., Goldbarg, A. \& Gullbrin, B. (1972). Fournal of Applied Physiology 33, 175-180.

Forgo, I. (1 980). Proceedings of the 3rd International Ginseng Symposium.

Gey, G. O., Cooper, K. H. \& Bottenberg, R. A. (1970). Fournal of American Medical Association $211,105$.

Girandola, R. N., Wiswell, R. A. \& Bulbulian, R. (1980). Biochemical Medicine 24, $218-222$.

Hallberg, L. \& Magnusson, B. (1984). Acta Medica Scandinavica $216,145-148$.

Haymes, E. M. (1980). Fournal of Drug Issues 10, 36I-369.

Herbert, V. (1979). American fournal of Clinical Nutrition 32, $1534^{-1} 54^{\circ}$.

Howald, H., Segesser, B. \& Körner, W. F. (1975). Annales of the New York Academy of Sciences $258,45^{8}-463$.

Hunding, A., Jordal, R. \& Paulev, P. E. (1981). Acta Medica Scandinavica 209, 315-318.

Karpuchina, Y. L., Oreshchenko, N. I. \& Stolyarova, N. A. (1967). Voprosy Pitaniya 26, 3.

Keren, G. \& Epstein, Y. (1980). Fournal of Sports Medicine 20, 145-148.

Kirchhoff, H. W. (1969). Nutrition et Dieta I 1, 184-192.

Lawrence, J. D., Bower, R. C., Riehl, W. P. \& Smith, J. L. (1975). American fournal of Clinical Nutrition 28, 205-208.

Magnusson, B., Hallberg, L., Rossander, L. \& Swolin, B. (1984a). Acta Medica Scandinavica $216,149-156$.

Magnusson, B., Hallberg, L., Rossander, L. \& Swolin, B. (1984b). Acta Medica Scandinavica 216, I $57-164$.

Marshall, F. N., Adamson, R. H. \& Long, J. P. (1961). Proceedings of the Society for Experimental Biology and Medicine 107, 420-422.

Nagawa, T., Kita, H., Aoki, J., Meashima, T. \& Shiozawa, K. (1968). Asian Medical fournal I r, $619-633$.

Namyslowski, L. (1980). Sportäratliche Praxis 13, i18-1 19.

Punnonen, R. \& Lukola, A. (1980). British Medical fournal 281, 11 10.

Rasch, P. J., Arnheim, D. D. \& Klafs, C. E. (1962). Sportärstliche Praxis 15, 10-1 3.

Sharman, I. M., Down, M. G. \& Norgan, N. G. (1976). Fournal of Sports Medicine 16, 21 5-225. 
Sharman, I. M., Down, M. G. \& Sen, R. N. (1971). British fournal of Nutrition 26, 265-276.

Shephard, R. J., Campbell, R., Pimm, P., Stuart, D. \& Wright, G. R. (1974). European fournal of Applied Physiology 33, 1 19-1 26.

Siegel, R. K. (1979). Fournal of the American Medical Association 241, 1614 16 r6.

Strauzenberg, S. E., Kässner, R., Böhm, R. \& Schneider, F. (198r). Medicine and Sports r4, $200-208$.

Sveriges Riksidrottsförbund (1982). Rapport frän RF:s kostgrupp. Stockholm: Sveriges Riksidrottsförbund.

Vellar, O. D. (1968). Scandinavian fournal of Clinical and Laboratory Investigation $2 \mathrm{I}$, $157-167$. 